STUDIO DELLA FLORA AUTOCTONA NELLA

BIOCONSERVAZIONE DEGLI ALIMENTI:

CARATTERIZZAZIONE TECNOLOGICA DI CEPPI ISOLATI

IN UN PRODOTTO IGP DELLA REGIONE MARCHE: IL

CIAUSCOLO

\title{
AUTHOCTHONOUS MICROFLORA IN BIOLOGICAL PRESERVATION OF FOODS: TECHNOLOGICAL CHARACTERIZATION OF STRAINS ISOLAT- ED FROM A PGI PRODUCT OF MARCHE REGION: CIAUSCOLO
}

\author{
Ciarrocchi F., Briscolini S., Lanciotti M., Striano G., Palombo B., Blasi G. \\ Istituto Zooprofilattico Sperimentale dell'Umbria e delle Marche - Fermo
}

SUMMARY

KEYWORDS

\begin{abstract}
The results of an investigation concerning the microbial population of Ciauscolo salami produced in the hinterland of the Marche Region, are reported. Phenotypic assays, namely inhibitory activity against Listeria monocytogenes, acidification ability, proteolysis, and lipolysis were performed. The technological characterization highlighted the presence of a high diversity of autochthonous bacterial population among the 14 producers investigated and a certain number of genetically different strains to be potentially used as starter cultures for the manufacture of Ciauscolo.
\end{abstract}

Ciauscolo, Lactic Acid Bacteria, Coagulase Negative Cocci, technological characterization
Il Ciauscolo è un insaccato fermentato tradizionale marchigiano, le cui metodiche di produzione, consolidate nel tempo, non prevedono l'impiego di colture starter. Come per altri prodotti tradizionali, l'importanza dello studio della microflora autoctona coinvolta nel processo produttivo risiede nella possibilità di valorizzare ulteriormente questo tipo di prodotto e al contempo, promuovere e approfondire il legame tra prodotto tipico, località e tecnologia di produzione. Questo studio si inserisce all' interno di un progetto di ricerca corrente finanziato dal Ministero della Salute: "Studio della flora autoctona nella bioconservazione degli alimenti: caratterizzazione di ceppi isolati in prodotti tradizionali della Regione Marche" (RC IZSUM 05/2007). L'obiettivo principale di questa ricerca ha riguardato lo studio della flora autoctona del Ciauscolo mediante l'impiego di tecniche innovative d'identificazione rapida di questi microrganismi e di valorizzazione del loro utilizzo, at- traverso lo studio delle attività biochimiche e antagoniste di interesse tecnologico. Vengono qui riportati i risultati della caratterizzazione tecnologica della microflora autoctona in ciauscoli di quattordici salumifici riconosciuti della Regione Marche. Settantadue ceppi di batteri lattici e 11 ceppi di cocchi coagulasi negativi, precedentemente identificati e biotipizzati mediante tecniche molecolari e provenienti sia da lotti di prodotto finito a 20 giorni di stagionatura (Ditte 1-14), sia da lotti campionati nelle diverse fasi di produzione (Ditta 1 ), sono stati sottoposti all'analisi dell' attività antagonistica nei confronti di cinque ceppi di Listeria monocytogenes mediante "Agar spot test" ed alla caratterizzazione tecnologica. Quest'ultima ha riguardato la valutazione delle attività: (i) acidificante dei LAB per via potenziometrica; (ii) proteolitica dei LAB e dei CCN sulla frazione sarcoplasmatica e miofibrillare delle proteine di carne suina mediante "Agar plate assay"; (iii) lipolitica dei 
CCN mediante "Agar plate assay" su Tributyrin agar. La selezione di poche specie dominanti nel prodotto finito ha evidenziato il ruolo giocato dalla flora lattica nel processo fermentativo di questo salume. La valutazione delle attività tecnologiche, nonché lo studio dell'attitudine dei ceppi selezionati alla bioconservazione dell'alimento mediante attività anti-Listeria, ha evidenziato diversi livelli di eterogeneità tecnologica, inter e intraspecifica, degli stipiti isolati, correlabile all' elevata eterogeneità genetica della popolazione microbica tipica del Ciauscolo. Cinquantadue dei 72 (circa 72\%) ceppi di LAB studiati hanno mostrato di avere attività antibatterica nei confronti di almeno un ceppo target, mediante la formazione di un alone di inibizione superiore ad $1 \mathrm{~mm}$. La maggiore attività antagonistica è stata osservata nei ceppi appartenenti alle specie Lactobacillus sakei, Pediococcus pentosaceus e Lactococcus lactis, con un alone di inibizione uguale o maggior di $3 \mathrm{~mm}$. In riferimento all' attività acidificante, è interessante notare come le due specie eterofermentanti facoltative Lactobacillus. plantarum e Lactobacillus. paraplantarum abbiano manifestato la migliore attitudine all'acidificazione, sia in termini di velocità, sia in termini di capacità acidificante totale. Non tutte le specie saggiate hanno mostrato capacità di idrolizzare le proteine della carne. E' infatti risultato positivo a tale caratteristica tecnologica solo il 50,3\% dei ceppi. Tutti i ceppi CCN saggiati hanno dimostrato di possedere attività lipolitica in seguito a semina su terreno agarizzato, ma come riscontrato per le altre attività tecnologiche studiate, sono stati osservati diversi livelli di intensità dell' attività lipolitica sia a livello di specie, sia a livello di stipite batterico. L'individuazione di alcuni ceppi dalle spiccate attitudini tecnologiche (L. sakei 48420-1 per l' attività anti-Listeria, Staphylococcus xylosus 48420-24 per l' attività lipolitica e le specie $L$. plantarum e L. paraplantarum per l' attività acidificante) suggerisce un possibile impiego di queste colture per la formulazione di colture starter autoctone a composizione mista, caratterizzate dalla coesistenza di più ceppi microbici, ciascuno con una differente attività tecnologica e/o bioconservativa che possano al contempo contribuire alla riduzione dell' appiattimento dell'aroma del prodotto apportato dall' industrializzazione del processo produttivo e dall' uso eccessivo di colture starter commerciali.

\section{BIBLIOGRAFIA}

15. Beccaceci A., Silvestri G., Santarelli S., Aquilanti L., Osimani A., Petruzzelli A., Clementi F. (2006). Investigation on lactic acid bacteria population of the fermented salami Ciauscolo by PCR-DGGE, Industria Conserve 81: 297-303.

16. Ciarrocchi F. Nardi S., Lanciotti M., Palombo B., Striano G., Venditti G., Blasi G. Definizione della biodiversità microbica nei "Ciauscoli" della Regione Marche. XII Congresso Nazionale S.I.Di.L.V. Genova, 2729/10/2010, pp. 183-184.

17. Drosinos H. E., Paramithiotis S., Kolovos G., Tsikouras I., Metaxopoulos I. (2007). Phenotypic and technological diversity of lactic acid bacteria and staphylococci isolated from traditionally fermented sausages in Southern Greece. Food Microb, 24: 260-270.

18. Essid I., Medini M., Hassouna M. (2009). Technological and safety properties of Lactobacillus plantarum strains isolated from a Tunisian traditional salted meat. Meat Sci, 81: 203-208.

19. Tomè E., Teixeira P., Gibbs P. A. (2006), Anti-listerial inhibitory lactic acid bacteria isolated from commercial cold smoked salmon. Food Micro, 23 (4): 399-405. 